\section{Patienten legen Wert auf multidisziplinäre Betreu- ung mit niedrigen Kosten}

\author{
Arslan IG et al. Patients', healthcare providers', \\ and insurance company employees' preferences \\ for knee and hip osteoarthritis care: a discrete \\ choice experiment. Osteoarthritis Cartilage \\ 2020; 28: 1316-1324
}

Die Behandlung von Knie- und Hüftgelenksarthrose (KHOA) fokussiert auf die Kontrolle der Symptome und die Verbesserung der Funktion. Trotz der Behandlungsmöglichkeiten wird nicht jeder Patient so behandelt, wie er nach den Leitlinien behandelt werden sollte und wie die persönlichen Präferenzen liegen. Arslan et al. ermittelten die Präferenzen von Patienten, Gesundheitsdienstleistern und Versicherungsgesellschaften für die Versorgung von KHOA.

Patienten, Gesundheitsdienstleister als auch die Mitarbeiter von Versicherungsgesellschaften bevorzugen in hohem Maße eine gemeinsame Konsultation durch Allgemeinmediziner und Orthopäden mit niedrigen Kosten aus eigener Tasche. Innerhalb der Patienten gibt es jedoch eine erhebliche Präferenzheterogenität. Die Forscher führten ein diskretes Entscheidungsexperiment durch, um einen Einblick in die Präferenzen der Studienteilnehmer zu gewinnen und herauszufinden, wie sie die Eigenschaften von verschiedenen Gesundheitseinrichtungen für KHOA bewerten und gegeneinander abwägen. In einem diskreten Entscheidungsexperiment werden die Teilnehmer wiederholt aufgefordert, eine Wahl zwischen verschiedenen hypothetischen Alternativen zu treffen, was schließlich ihre Präferenzen offenbart. Die Experten stellten Patienten, Gesundheitsdienstleistern und Mitarbeitern von Versicherungsgesellschaften in einem Fragebogen mehrere Alternativen einer KHOA-Betreuung mit verschiedenen Kombinationen von Attributen (z. B. Wartezeit auf einen Termin, Höhe von Kosten bei einer Eigenbeteiligung, Erreichbarkeit der KHOAInstitution, usw.) vor und baten diese, die persönlichen Präferenzen anzugeben. Die Attribute wählten die Forscher vorab nach einer Befragung von Experten aus der KHOAVersorgung und KHOA-Patienten aus. Neben dem Fragebogen erhoben die Wissenschaftler bei den Patienten außerdem Daten zur Demografie (z. B. Geschlecht, Alter, Beschäftigungsstatus), zu gesundheitsbezogenen Fragen (Dauer der Knie-/ Hüftbeschwerden, Schmerzen, Grad der Verletzung, Lebensqualität) und zu Erfahrungen mit der Gesundheitsversorgung. Die Fragebögen für Gesundheitsdienstleister und Mitarbeiter von Versicherungsgesellschaften enthielten demografische Fragen und arbeitsbezogene Fragen (z. B. Beruf und Bildungsniveau). Die Teilnehmer rekrutierten die Forscher über einen kommerziellen Anbieter von Umfragestichproben (Dynata).

Die Wissenschaftler schlossen 648 KHOAPatienten, 76 Gesundheitsdienstleister und 150 Mitarbeiter von Versicherungsgesellschaften in die Analyse ein. Die Forscher identifizierten 6 wichtige Attribute: Gesundheitsversorgung mit geringer Kostenbeteiligung, gemeinsame Konsultation von Hausarzt und Orthopäde, lange Konsultation, direkter Zugang zu Spezial-Equipment, kurze Anfahrtswege und kurze Wartezeiten. Eine niedrige Selbstbeteiligung erwies sich für Patienten und Gesundheitsdienstleister im Vergleich zu allen anderen Attributen am wichtigsten. Im Gegensatz hierzu erachteten die Mitarbeiter der Krankenkassen eine gemeinsame Beratung durch Allgemeinmediziner und Orthopä- 
den am wichtigsten. Die Dauer der Konsultation war für Patienten und Beschäftigte der Krankenkasse am unwichtigsten, während für die Leistungserbringer im Gesundheitswesen die Wartezeiten am unwichtigsten waren. Eine latente Klassenanalyse zeigte, dass KHOA-Patienten ohne Gelenkersatz zu einer Gesundheitsversorgung mit niedrigen Kosten aus eigener Tasche tendieren. Patienten mit einem Gelenkersatz und/oder geringer krankheitsspezifischer Lebensqualität tendierten hingegen zu einer Versorgung durch einen Orthopäden. Patienten, die bereits wegen Knieoder Hüftproblemen behandelt wurden, bevorzugen eher eine gemeinsame Beratung durch einen Allgemeinmediziner und einen Orthopäden sowie einen direkten Zugang zu Spezialgeräten.

\section{FAZIT}

Patienten mit Knie- und Hüftgelenksarthrose (KHOA), Gesundheitsdienstleister im KHOA-Sektor als auch Versicherungsangestellte legen im Rahmen der medizinischen Betreuung viel Wert auf eine gemeinsame Konsultation von Allgemeinmedizinern und Orthopäden und auf eine niedrige Selbstbeteiligung an den Kosten der Behandlung. Die Ergebnisse dieser Studie können helfen, durch eine Auswahl passender Attribute eine stärker individualisierte Gesundheitsversorgung im KHOA-Sektor zu etablieren, so die Autoren.

Dr. Maddalena Angela Di Lellis, Tübingen 